Scattered field generation and optical forces in transformation optics

This content has been downloaded from IOPscience. Please scroll down to see the full text. 2016 J. Opt. 18044021

(http://iopscience.iop.org/2040-8986/18/4/044021)

View the table of contents for this issue, or go to the journal homepage for more

Download details:

IP Address: 93.84.43.170

This content was downloaded on 01/04/2016 at $16: 52$

Please note that terms and conditions apply. 


\title{
Scattered field generation and optical forces in transformation optics
}

\author{
A V Novitsky \\ Department of Theoretical Physics and Astrophysics, Belarusian State University, Nezavisimosti Ave. 4 , \\ 220030 Minsk, Belarus \\ E-mail: andrey.novitsky@tut.by \\ Received 13 August 2015, revised 10 September 2015 \\ Accepted for publication 11 September 2015 \\ Published 1 April 2016

\begin{abstract}
In this paper we develop an approach for making various scattered electromagnetic fields on the transformation-optics ground. To do so, we use the a special coordinate transformation from the a vacuum virtual space to physical space, which changes the boundary of the scattering device upon transformation. We explore this approach for small scatterers compared with radiation wavelength, which allows us to predict the arbitrarily directed optical forces. Obtaining scattered fields and optical forces can be useful in nano-optics and optical micromanipulation.
\end{abstract}

Keywords: light scattering, optical force, transformation optics, metamaterials

\section{Introduction}

Transformation optics is used to predict the material parameters of devices with preassigned field distributions [1-4]. In the case of cloaks [5-8] the scattered field and the field inside a hidden cavity are absent. Rotator $[9,10]$ performs the field rotation by a certain angle. Illusion devices [11-13] make the desired scattered fields, while a transformationoptics lens [14] creates a special field distribution on the output having a plane wave on the input. Most of the above applications can be clearly obtained using the coordinate transformation from the virtual (electromagnetic) space to the real (physical) space. The transformation region of the device is allocated in the virtual space, which is subjected to coordinate transformation. The remainder of the virtual space does not change; therefore, the material parameters and the fields do not change, either.

Properly designed electromagnetic fields have important practical applications; consequently, they are an increasingly popular focus of study. Directional light scattering caused by the interference of electric and magnetic dipoles have applications for the light-on-chip nanoantennas [15-18]. Primary forward- and backward-scattering can create efficient interaction between light and particle and thus in the strong optical forces required for stable microparticle manipulation [19-21]. Since transformation optics is intended for novel applications, we expect a significant demand for these unusual scattered fields and optical forces.
In this paper, we use the field approach in transformation optics developed by Novitsky et al (2012) and Novitsky (2011) [14, 22]. It is advantageous, when we need to deal with the fields themselves, to find a distinguished field distribution. This approach involves writing the general solutions in transformation media and connecting them with the fields outside using the boundary conditions between the media. In Novitsky et al (2012) [14], one can find the applications of the field approach to the cloaking devices, concentrators, and rotators. The general solutions can be expressed in terms of the so-called potential functions $\psi_{i}(\boldsymbol{r})$ $(i=1,2,3)$ setting the inverse Jacobian matrix $J$ of the transformation between the virtual $\boldsymbol{r}^{\prime}(\boldsymbol{r})=\sum_{j=1}^{3} \psi_{j}(\boldsymbol{r}) \boldsymbol{n}_{i}$ and physical $\boldsymbol{r}$ spaces as

$J^{-1}=\nabla \otimes \boldsymbol{r}^{\prime}(\boldsymbol{r})=\nabla \psi_{1} \otimes \boldsymbol{n}_{1}+\nabla \psi_{2} \otimes \boldsymbol{n}_{2}+\nabla \psi_{3} \otimes \boldsymbol{n}_{3}$,

where $\boldsymbol{n}_{i}$ are the unit orthogonal vectors and $\boldsymbol{a} \otimes \boldsymbol{b}$ is the outer product of vectors $\boldsymbol{a}$ and $\boldsymbol{b}$. Potential functions are often the coordinates in virtual space $x_{i}^{\prime}$, but they can have more general meaning as the complex-valued functions of the circular frequency $\omega$. Taking the inverse matrix, we obtain the Jacobian matrix in the form

$$
J=\frac{\sum_{i=1}^{3} \boldsymbol{n}_{i} \otimes \boldsymbol{a}_{i}}{\nabla \psi_{1}\left(\nabla \psi_{2} \times \nabla \psi_{3}\right)},
$$


where $\times$ denotes the vector product,

$\boldsymbol{a}_{1}=\nabla \psi_{2} \times \nabla \psi_{3}=\nabla \times\left(\psi_{2} \nabla \psi_{3}\right)$,

$\boldsymbol{a}_{2}=\nabla \psi_{3} \times \nabla \psi_{1}=\nabla \times\left(\psi_{3} \nabla \psi_{1}\right)$

and

$\boldsymbol{a}_{3}=\nabla \psi_{1} \times \nabla \psi_{2}=\nabla \times\left(\psi_{1} \nabla \psi_{2}\right)$.

The fields $\boldsymbol{E}^{\prime}$ and $\boldsymbol{H}^{\prime}$ in the medium with permittivity $\hat{\varepsilon}^{\prime}$ and permeability $\hat{\mu}^{\prime}$ are solutions of the Maxwell equations in the virtual space (note that they should not necessarily coincide with the fields and material parameters outside the transformation medium). The fields in the physical space are equal to

$$
\begin{gathered}
\boldsymbol{E}(\boldsymbol{r})=J^{-1} \boldsymbol{E}^{\prime}\left(\boldsymbol{r}^{\prime}\right)=\sum_{i=1}^{3} \nabla \psi_{i}\left(\boldsymbol{n}_{i} \boldsymbol{E}^{\prime}\right), \\
\boldsymbol{H}(\boldsymbol{r})=J^{-1} \boldsymbol{H}^{\prime}\left(\boldsymbol{r}^{\prime}\right)=\sum_{i=1}^{3} \nabla \psi_{i}\left(\boldsymbol{n}_{i} \boldsymbol{H}^{\prime}\right) .
\end{gathered}
$$

The permittivity and permeability tensors in the physical space read

$$
\begin{gathered}
\hat{\varepsilon}=\frac{J^{T} \hat{\varepsilon}^{\prime} J}{\operatorname{det}(J)}=\frac{\sum_{i, j=1}^{3} \varepsilon_{i j}^{\prime} \boldsymbol{a}_{i} \otimes \boldsymbol{a}_{j}}{\nabla \psi_{1}\left(\nabla \psi_{2} \times \nabla \psi_{3}\right)}, \\
\hat{\mu}=\frac{J^{T} \hat{\mu}^{\prime} J}{\operatorname{det}(J)}=\frac{\sum_{i, j=1}^{3} \mu_{i j}^{\prime} \boldsymbol{a}_{i} \otimes \boldsymbol{a}_{j}}{\nabla \psi_{1}\left(\nabla \psi_{2} \times \nabla \psi_{3}\right)},
\end{gathered}
$$

where $\operatorname{det}(J)=1 / \nabla \psi_{1}\left(\nabla \psi_{2} \times \nabla \psi_{3}\right), \quad \varepsilon_{i j}^{\prime}=\boldsymbol{n}_{i} \hat{\varepsilon}^{\prime} \boldsymbol{n}_{j}$, and $\mu_{i j}^{\prime}=\boldsymbol{n}_{i} \hat{\mu}^{\prime} \boldsymbol{n}_{j}$. Equations (3) and (4) coincide with those used in the conventional theory of transformation optics [4].

The paper consists of 5 sections-including the introduction. In section 2, we derive the expressions for an optical force in terms of the fields in a transformation medium and in terms of the potential functions. In section 3, we discuss the general idea of generating scattered fields and apply it to our investigation, in which the particle made of transformation medium is much smaller than the radiation wavelength. We examine the closed-form results - comparing them to the fullwave simulation. In section 4 , we describe how to create arbitrarily directed optical forces. In section 5, we conclude the paper and discuss the further directions for study of the control of scattered fields and optical forces.

\section{Transformation optics of the optical force}

The expression for the optical force follows from the conservation-of-momentum equation [23]

$$
\frac{\partial \boldsymbol{g}}{\partial t}-\nabla \hat{T}=-\boldsymbol{f}_{L}
$$

where $\boldsymbol{g}=\boldsymbol{S} / c^{2}$ is the field momentum, $\boldsymbol{S}$ is the Poynting vector, $\hat{T}$ is the Maxwell stress tensor, and $f_{L}$ is the Lorentz force density. By integrating this equation over time within the period of oscillation $T$, one obtains zero contribution of the field momentum $g$. Integrating over the volume embracing the particle, we derive the time-averaged optical force

$$
\boldsymbol{F}=\frac{1}{T} \int_{0}^{T} \mathrm{~d} t \int_{V} \nabla \hat{T} \mathrm{~d} V=\int_{S}\langle\hat{T}\rangle \mathrm{d} \boldsymbol{s},
$$

where the time-averaged Maxwell stress tensor reads

$$
\begin{aligned}
\langle\hat{T}\rangle= & \frac{1}{T} \int_{0}^{T} \nabla \hat{T} \mathrm{~d} t=\frac{1}{8 \pi} \operatorname{Re}\left[\boldsymbol{E}^{(\mathrm{t})} \otimes \boldsymbol{E}^{(\mathrm{t}) *}\right. \\
& \left.+\boldsymbol{H}^{(\mathrm{t})} \otimes \boldsymbol{H}^{(\mathrm{t}) *}-\frac{1}{2}\left(\left|\boldsymbol{E}^{(\mathrm{t})}\right|^{2}+\left|\boldsymbol{H}^{(\mathrm{t})}\right|^{2}\right) \mathbf{1}\right] .
\end{aligned}
$$

Here $\mathbf{1}$ is the unit three-dimensional tensor, $S$ is any surface around the particle, and ${ }^{*}$ is the complex conjugate. Total electric and magnetic fields outside the scatterer equal the superpositions of the incident and scattered fields as $\boldsymbol{E}^{(\mathrm{t})}=\boldsymbol{E}^{(\mathrm{inc})}+\boldsymbol{E}^{(\mathrm{sc})}$ and $\boldsymbol{H}^{(\mathrm{t})}=\boldsymbol{H}^{(\mathrm{inc})}+\boldsymbol{H}^{(\mathrm{sc})}$.

Although the force is usually written by means of the incident and scattered electromagnetic fields, it can be rewritten in terms of the field inside the scatterers $\boldsymbol{E}$ and $\boldsymbol{H}$. In fact, the tangential field strengths and normal field inductions at the interface between the scatterer and the ambient medium $S_{\mathrm{sc}}$ are continuous: $\left.\quad \boldsymbol{I} \boldsymbol{E}^{(\mathrm{t})}\right|_{S_{\mathrm{sc}}}=\left.\boldsymbol{I} \boldsymbol{E}\right|_{S_{\mathrm{sc}}},\left.\quad \boldsymbol{I} \boldsymbol{H}^{(\mathrm{t})}\right|_{S_{\mathrm{sc}}}=\left.\boldsymbol{I} \boldsymbol{H}\right|_{S_{\mathrm{sc}}}$, $\left.E_{n}^{(\mathrm{t})}\right|_{S_{\mathrm{sc}}}=\left.\boldsymbol{n} \varepsilon \boldsymbol{E}\right|_{S_{\mathrm{sc}}}$, and $\left.H_{n}^{(\mathrm{t})}\right|_{S_{\mathrm{sc}}}=\left.\boldsymbol{n} \mu \boldsymbol{H}\right|_{S_{\mathrm{sc}}}$, where $\boldsymbol{n}$ is a unit vector normal to the interface, and $I=\mathbf{1}-\boldsymbol{n} \otimes \boldsymbol{n}$ is the projector onto the interface. If the surface of integration $S$ coincides with the interface between the particle and the ambient medium $S_{\mathrm{sc}}$, then we can replace the total electromagnetic field with the field inside the particle.

Then in terms of the potential functions $\psi_{i}$ (see (3) and (4)) we write

$$
\begin{gathered}
\left.\boldsymbol{E}^{(\mathrm{t})}\right|_{S_{\mathrm{sc}}}=\left.\sum_{i, j=1}^{3}\left(I \nabla \psi_{i} \delta_{i j}+\varepsilon_{i j}^{\prime} \boldsymbol{n}\left(\boldsymbol{n} \boldsymbol{a}_{i}\right)\right) E_{j}^{\prime}\right|_{S_{\mathrm{sc}}}, \\
\left.\boldsymbol{H}^{(\mathrm{t})}\right|_{S_{\mathrm{sc}}}=\left.\sum_{i, j=1}^{3}\left(I \nabla \psi_{i} \delta_{i j}+\mu_{i j}^{\prime} \boldsymbol{n}\left(\boldsymbol{n} \boldsymbol{a}_{i}\right)\right) H_{j}^{\prime}\right|_{S_{\mathrm{sc}}} .
\end{gathered}
$$

where $\delta_{i j}$ is the Kronecker delta.

Let an electromagnetic wave propagates in the empty virtual space $\left(\varepsilon^{\prime}=1\right.$ and $\left.\mu^{\prime}=1\right)$, the fields being represented as $\boldsymbol{E}^{\prime}=A \exp \left(\mathrm{i} k_{0} x^{\prime}\right) \boldsymbol{n}_{2}$ and $\boldsymbol{H}^{\prime}=A \exp \left(\mathrm{i} k_{0} x^{\prime}\right) \boldsymbol{n}_{3}$, where $k_{0}=\omega / c$ is the vacuum wavenumber and $A$ is the amplitude. Passing through a closed, empty region in the empty virtual space, the wave does not scatter. Transformation optics allows us to find the fields in physical space using (3): $\boldsymbol{E}=A \exp \left(\mathrm{i} k_{0} \psi_{1}\right) \nabla \psi_{2}$ and $\boldsymbol{H}=A \exp \left(\mathrm{i} k_{0} \psi_{1}\right) \nabla \psi_{3}$, where $x^{\prime}=\psi_{1}$ (we assume the potential functions are the coordinates in the virtual space $\psi_{i}=x_{i}^{\prime}$, therefore real-valued functions). Hence $\left.I \boldsymbol{E}^{(\mathrm{t})}\right|_{S_{\mathrm{sc}}}=\left.A \exp \left(\mathrm{i} k_{0} \psi_{1}\right) I \nabla \psi_{2}\right|_{S_{\mathrm{sc}}}$ and $\left.E_{n}^{(\mathrm{t})}\right|_{S_{\mathrm{sc}}}=\left.A \exp \left(\mathrm{i} k_{0} \psi_{1}\right)\left[\boldsymbol{n}\left(\nabla \psi_{3} \times \nabla \psi_{1}\right)\right]\right|_{S_{\mathrm{sc}}}$. Total electric and magnetic fields at the interface between the scatterer and the ambient medium read

$$
\begin{aligned}
& \left.\boldsymbol{E}^{(\mathrm{t})}\right|_{S_{\mathrm{sc}}}=\left.A \mathrm{e}^{\mathrm{i} k_{0} \psi_{1}}\left(I \nabla \psi_{2}+\boldsymbol{n}\left[\boldsymbol{n}\left(\nabla \psi_{3} \times \nabla \psi_{1}\right)\right]\right)\right|_{S_{\mathrm{sc}}}, \\
& \left.\boldsymbol{H}^{(\mathrm{t})}\right|_{S_{\mathrm{sc}}}=\left.A \mathrm{e}^{\mathrm{i} k_{0} \psi_{1}}\left(I \nabla \psi_{3}+\boldsymbol{n}\left[\boldsymbol{n}\left(\nabla \psi_{1} \times \nabla \psi_{2}\right)\right]\right)\right|_{S_{\mathrm{sc}}} .
\end{aligned}
$$

By substituting (9) into (6) and remembering that $\mathrm{d} \boldsymbol{s}=\boldsymbol{n} \mathrm{d} s$, we derive the optical force expressed via the real 
potential functions $\psi_{i}$ as

$$
\begin{aligned}
\boldsymbol{F}= & \frac{A^{2}}{8 \pi} \int_{S_{\mathrm{sc}}}\left\{\left[\boldsymbol{n}\left(\nabla \psi_{3} \times \nabla \psi_{1}\right)\right] I \nabla \psi_{2}\right. \\
& +\left[\boldsymbol{n}\left(\nabla \psi_{1} \times \nabla \psi_{2}\right)\right] I \nabla \psi_{3} \\
& -\frac{\boldsymbol{n}}{2}\left(\left|I \nabla \psi_{2}\right|^{2}+\left|I \nabla \psi_{3}\right|^{2}-\left[\boldsymbol{n}\left(\nabla \psi_{3} \times \nabla \psi_{1}\right)\right]^{2}\right. \\
& \left.\left.-\left[\boldsymbol{n}\left(\nabla \psi_{1} \times \nabla \psi_{2}\right)\right]^{2}\right)\right\} \mathrm{d} s .
\end{aligned}
$$

Coordinate transformation $x_{i}^{\prime}(\boldsymbol{r})=\psi_{i}(\boldsymbol{r})$ should satisfy the boundary conditions at the interface between the particle and ambient medium. When the outer interface of the transformation medium $S_{\mathrm{sc}}$ does not change upon transformation $\left(\left.\psi_{i}\right|_{S_{\mathrm{sc}}}=\left.x_{i}\right|_{S_{\mathrm{sc}}}\right)$, the fields out of the transformation medium coincide with those in the virtual space. If the boundary between the ambient and transformation media changes, as in the case of the illusion devices $[11,13]$, the two-shell transformation medium is used: the inner transformation medium replaces the scatterer itself, while the outer one keeps condition $\left.\psi_{i}\right|_{S_{\mathrm{sc}}}=\left.x_{i}\right|_{S_{\mathrm{sc}}}$ after transformation.

Thus if one knows the field in the virtual space, it is possible to write the force in terms of potential functions (or Jacobian) without knowing the scattered fields. Unfortunately, in such a formulation, the force described by the potential functions has no heuristic power. Indeed, the force does not change upon the transformation. Hence it can be even simpler to perform calculations in the auxiliary virtual space, in which we know the fields $\boldsymbol{E}^{\prime}$ and $\boldsymbol{H}^{\prime}$. Only if we determine the potential functions from the boundary conditions specifying the incident and scattered fields we can anticipate a different value of the force compared with the conventional transformation technique. In this case, the interface $S_{\mathrm{sc}}$ is different in virtual and physical spaces and the relationship $\left.\psi_{i}\right|_{S_{\mathrm{sc}}}=\left.x_{i}\right|_{S_{\mathrm{sc}}}$ no longer holds true.

\section{Scattered field engineering}

The goal of this section is to demonstrate that the transformation optics can be adopted for the generation of the demanded scattered fields. First, we will describe the general theory and then we will apply it to the formation of quasistatic electric fields. Scattered field is induced by the incident field and linearly depends on it at the interface between the scatterer and ambient medium. Field inside the scatterer can be written by means of auxiliary fields $\boldsymbol{E}^{\prime}$ and $\boldsymbol{H}^{\prime}$, satisfying the Maxwell equations in the virtual space characterized by $\hat{\varepsilon}^{\prime}$ and $\hat{\mu}^{\prime}$.
The boundary conditions at the scatterer surface stem from the continuity of the tangential field components:

$$
\begin{gathered}
\left.I\left(\boldsymbol{E}^{(\mathrm{inc})}+\boldsymbol{E}^{(\mathrm{sc})}\right)\right|_{S_{\mathrm{sc}}}=\left.I \sum_{i=1}^{3} \nabla \psi_{i}\left(\boldsymbol{n}_{i} \boldsymbol{E}^{\prime}\right)\right|_{S_{\mathrm{sc}}}, \\
\left.I\left(\boldsymbol{H}^{(\mathrm{inc})}+\boldsymbol{H}^{(\mathrm{sc})}\right)\right|_{S_{\mathrm{sc}}}=\left.I \sum_{i=1}^{3} \nabla \psi_{i}\left(\boldsymbol{n}_{i} \boldsymbol{H}^{\prime}\right)\right|_{S_{\mathrm{sc}}} .
\end{gathered}
$$

Using some scattered fields satisfying Maxwell's equations, equation (11) yields the system of differential equations for the potential functions $\psi_{i}$. If the potential functions exist, the presumed scattered electromagnetic field appears as a result of the scattering by a particle with the permittivity and permeability described according to (4). The optical force can be calculated using either the incident and scattered fields (6) or potential functions (equation (10) or similar).

The scattered field can be quite complicated, but in general it is the multipole expansion. When the particle size is small enough, the series can be truncated up to dipoles, quadrupoles, etc. Thus, we can manipulate the scattered fields and optical forces using the transformation optics.

Let us apply the proposed idea to the particular case of the incident plane wave (fields $\boldsymbol{E}^{(\mathrm{inc})}=A \exp \left(\mathrm{i} k_{0} x\right) \boldsymbol{e}_{y}$ and $\left.\boldsymbol{H}^{(\text {inc })}=A \exp \left(\mathrm{i} k_{0} x\right) \boldsymbol{e}_{z}\right)$ generating a rotated dipolar scattering pattern. Assuming the particle size is much smaller than the radiation wavelength, we represent the scattered electric and magnetic fields of the electric dipole at the interface $S_{\mathrm{sc}}$ in the form

$$
\left.\left.\boldsymbol{E}^{(\mathrm{sc})}\right|_{S_{\mathrm{sc}}} \approx \hat{R} \boldsymbol{E}_{0}^{(\mathrm{sc})}\right|_{S_{\mathrm{sc}}},\left.\quad \boldsymbol{H}^{(\mathrm{sc})}\right|_{S_{\mathrm{sc}}} \approx 0
$$

where $\hat{R}$ is the three-dimensional rotation matrix and $\left.\boldsymbol{E}_{0}^{(\mathrm{sc})}\right|_{S_{\mathrm{sc}}}$ is the scattered field without rotation. For the small particle size the scattered field is approximately the near field of the point dipole $\boldsymbol{p}=\hat{\alpha}_{e} \boldsymbol{E}^{(\mathrm{inc})}(0)$ :

$$
\left.\boldsymbol{E}^{(\mathrm{sc})}\right|_{S_{\mathrm{sc}}}=\left.\hat{R} \frac{3 \boldsymbol{r} \otimes \boldsymbol{r}-|\boldsymbol{r}|^{2} \mathbf{1}}{|\boldsymbol{r}|^{5}} \hat{\alpha}_{e} \boldsymbol{E}^{(\mathrm{inc})}(0)\right|_{S_{\mathrm{sc}}},
$$

where $\hat{\alpha}_{e}$ is particle's polarizability tensor and $\boldsymbol{E}^{(\mathrm{inc})}(0)$ is the incident electric field at the center of the particle.

The field in the transformation medium can be written in terms of the field in the virtual space $\boldsymbol{E}^{\prime}=A^{\prime} \exp \left(\mathrm{i} k_{0} \sqrt{\varepsilon^{\prime} \mu^{\prime}} x^{\prime}\right) \boldsymbol{n}_{2}$ and $\boldsymbol{H}^{\prime}=A^{\prime} \sqrt{\varepsilon^{\prime} / \mu^{\prime}}$ $\exp \left(\mathrm{i} k_{0} \sqrt{\varepsilon^{\prime} \mu^{\prime}} x^{\prime}\right) \boldsymbol{n}_{3}$ as

$$
\boldsymbol{E}=A^{\prime} \mathrm{e}^{\mathrm{i} k_{0} \sqrt{\varepsilon^{\prime} \mu^{\prime}}} \psi_{1} \nabla \psi_{2}, \quad \boldsymbol{H}=A^{\prime} \sqrt{\frac{\varepsilon^{\prime}}{\mu^{\prime}}} \mathrm{e}^{\mathrm{i} k_{0} \sqrt{\varepsilon^{\prime} \mu^{\prime}} \psi_{1}} \nabla \psi_{3} .
$$


The boundary conditions read

$$
\begin{aligned}
& I\left(A \mathrm{e}^{\mathrm{i}} k_{0} x \boldsymbol{e}_{y}+A \hat{R} \frac{3 \boldsymbol{r} \otimes \boldsymbol{r}-|\boldsymbol{r}|^{2} \mathbf{1}}{|\boldsymbol{r}|^{5}} \hat{\alpha}_{e} \boldsymbol{e}_{y}\right)_{S_{\mathrm{sc}}}
\end{aligned}
$$

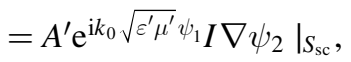

$$
\begin{aligned}
& \left.I A \mathrm{e}^{\mathrm{i} k_{0} x} \boldsymbol{e}_{z}\right|_{S_{\text {sc }}}=\left.A^{\prime} \sqrt{\frac{\varepsilon^{\prime}}{\mu^{\prime}}} \mathrm{e}^{\mathrm{i} k_{0} \sqrt{\varepsilon^{\prime} \mu^{\prime}}} \psi_{1} I \nabla \psi_{3}\right|_{S_{\text {sc }}} .
\end{aligned}
$$

The equation of equal phases puts restriction on the potential function $\psi_{1}$ as

$$
\left.\psi_{1}\right|_{S_{\mathrm{sc}}}=\left.\frac{1}{\sqrt{\varepsilon^{\prime} \mu^{\prime}}} x\right|_{S_{\mathrm{sc}}} .
$$

Then from the second equation in (15) one obtains the third potential function

$$
\left.\psi_{3}\right|_{S_{\mathrm{sc}}}=\left.\frac{A}{A^{\prime}} \sqrt{\frac{\mu^{\prime}}{\varepsilon^{\prime}}} z\right|_{S_{\mathrm{sc}}} .
$$

Function $\psi_{2}$ should be determined from the first equation in (15), where we neglect the phase within the point scatterer:

$$
A I\left(\boldsymbol{e}_{y}+\hat{R} \frac{3 \boldsymbol{r} \otimes \boldsymbol{r}-|\boldsymbol{r}|^{2} 1}{|\boldsymbol{r}|^{5}} \hat{\alpha}_{e} \boldsymbol{e}_{y}\right)_{S_{\mathrm{sc}}}=\left.A^{\prime} I \nabla \psi_{2}\right|_{S_{\mathrm{sc}}} .
$$

We cannot solve this equation before specifying the form of the particle. It should be noted that the potential function $\psi_{1}$ can be chosen in another way, as long as the phase is negligible: $\left.\sqrt{\varepsilon^{\prime} \mu^{\prime}} k_{0} \psi_{1}\right|_{S_{\mathrm{sc}}} \sim k_{0} a \approx 0$.

Further we consider an infinitely long cylindrical particle with the circular cross-section of radius $a$ in the plane $(x, y)$. The incident plane wave propagates along the $x$ axis. Dipole response of the cylinder is the superposition of the responses of the point dipolar moments distributed along the axis $z$. Treating the dipoles as static quantities, we derive

$$
\left.\boldsymbol{E}^{(\mathrm{sc})}\right|_{S_{\mathrm{sc}}}=\left.\hat{R} \frac{2\left(\boldsymbol{e}_{\rho} \otimes \boldsymbol{e}_{\rho}-\boldsymbol{e}_{\varphi} \otimes \boldsymbol{e}_{\varphi}\right)}{\rho^{2}} \hat{\alpha}_{e} \boldsymbol{E}^{(\mathrm{inc})}(0)\right|_{S_{\mathrm{sc}}},
$$

where $\rho, \varphi$, and $z$ are the cylindrical coordinates, $\boldsymbol{e}_{\rho}, \boldsymbol{e}_{\varphi}$, and $\boldsymbol{e}_{\mathrm{z}}$ are the basis vectors. Since only the scattered electric field changes after turning to the cylindrical geometry, potential functions $\psi_{1}$ and $\psi_{3}$ are given by (16) and (17): $\psi_{1}(a, \varphi, z)=a \cos \varphi / \sqrt{\varepsilon^{\prime} \mu^{\prime}}$ and $\psi_{3}(a, \varphi, z)=\sqrt{\mu^{\prime} / \varepsilon^{\prime}} z$, where $A=A^{\prime}$ is used. The differential equation for $\psi_{2}$ reads

$$
I\left(\boldsymbol{e}_{y}+\hat{R} \frac{2\left(\boldsymbol{e}_{\rho} \otimes \boldsymbol{e}_{\rho}-\boldsymbol{e}_{\varphi} \otimes \boldsymbol{e}_{\varphi}\right)}{\rho^{2}} \hat{\alpha}_{e} \boldsymbol{e}_{y}\right)_{\rho=a}=\left.I \nabla \psi_{2}\right|_{\rho=a} .
$$

The normal vector to cylinder's surface and projector are $\boldsymbol{n}=\boldsymbol{e}_{\rho}$ and $I=\mathbf{1}-\boldsymbol{e}_{\rho} \otimes \boldsymbol{e}_{\rho}$, respectively. We take the scalar polarizability in the cylinder cross-section $\alpha_{e}$ and consider the rotation of the scattering pattern in the plane $(x, y)$. Thus the rotation is performed with respect to the axis $z$ :

$$
\hat{R}=\cos \beta\left(\mathbf{1}-\boldsymbol{e}_{z} \otimes \boldsymbol{e}_{z}\right)+\sin \beta \boldsymbol{e}_{z}^{\times}+\boldsymbol{e}_{z} \otimes \boldsymbol{e}_{z},
$$

where $\beta$ is the rotation angle. Solution of the equation for $\psi_{2}$

$$
\frac{1}{a} \frac{\partial \psi_{2}}{\partial \varphi} \boldsymbol{e}_{\varphi}+\frac{\partial \psi_{2}}{\partial z} \boldsymbol{e}_{z}=\left(\cos \varphi-\frac{2 \alpha_{e}}{a^{2}} \cos (\varphi+\beta)\right) \boldsymbol{e}_{\varphi}
$$

is equal to

$$
\psi_{2}(a, \varphi, z)=a\left(\sin \varphi-\frac{2 \alpha_{e}}{a^{2}} \sin (\varphi+\beta)\right) .
$$

The simplest potential functions resulting in the rotation of the scattering pattern are of the form

$$
\begin{aligned}
& \psi_{1}(\boldsymbol{r})=\frac{r \cos \varphi}{\sqrt{\varepsilon^{\prime} \mu^{\prime}}}, \psi_{2}(\boldsymbol{r})=r\left(\sin \varphi-\frac{2 \alpha_{e}}{a^{2}} \sin (\varphi+\beta)\right), \\
& \psi_{3}(\boldsymbol{r})=\sqrt{\frac{\mu^{\prime}}{\varepsilon^{\prime}}} z .
\end{aligned}
$$

When the plane wave is scattered by the cylinder, we can expect the scattered electric field in the near-field zone is quasistatic and equals

$$
\begin{aligned}
\boldsymbol{E}^{(\mathrm{sc})} & =\frac{2 \alpha_{e} A}{\rho^{2}} \hat{R}\left(\boldsymbol{e}_{\rho} \otimes \boldsymbol{e}_{\rho}-\boldsymbol{e}_{\varphi} \otimes \boldsymbol{e}_{\varphi}\right) \boldsymbol{e}_{y} \\
& =\frac{2 \alpha_{e}}{\rho^{2}}\left(\sin (\varphi+\beta) \boldsymbol{e}_{\rho}-\cos (\varphi+\beta) \boldsymbol{e}_{\varphi}\right)
\end{aligned}
$$

with the $x$-component of the field

$$
E_{x}^{(\mathrm{sc})}=\frac{2 \alpha_{e} A}{\rho^{2}} \sin (2 \varphi+\beta) .
$$

It takes maximum values in the directions specified by the angles $\varphi_{1}=\pi / 4-\beta / 2$ and $\varphi_{2}=5 \pi / 4-\beta / 2$ and minimum values at $\varphi_{3}=3 \pi / 4-\beta / 2$ and $\varphi_{4}=7 \pi / 4-\beta / 2$.

In figure 1 , we show the result of the COMSOL simulation of the $x$-component of the scattered electric field. The cylinder's material parameters are calculated using (4) for the potential functions $\psi_{i}$ given by (24). One notices quite good correspondence with the predicted scattered field. Discrepancies are caused by the approximations used in the model. It is interesting that the elliptical region in the virtual space is transformed into the circular one in the physical space, in contrast to the usual result in which the transformation region remains unaltered. This fact should not be surprising, because the common continuity of the coordinates in virtual and physical spaces is replaced by the continuity of the tangential fields. The continuity of the fields is a more generic feature, having the continuity of the coordinates as a particular case of the absence of additional scattered fields compared with those in the virtual space.

If the angle distribution of the scattered field is arbitrary, the potential function $\psi_{i}$ can be also found. In this case, we specify not the scattered field itself, but only the scattered field at the boundary $\rho=a$. Spatial evolution of the scattered fields away of the boundary should satisfy the Maxwell equations, but this is immaterial. It can be understood as follows. In the quasistatic approximation, we still have nearzero magnetic field; therefore, the changes of the magnetic 


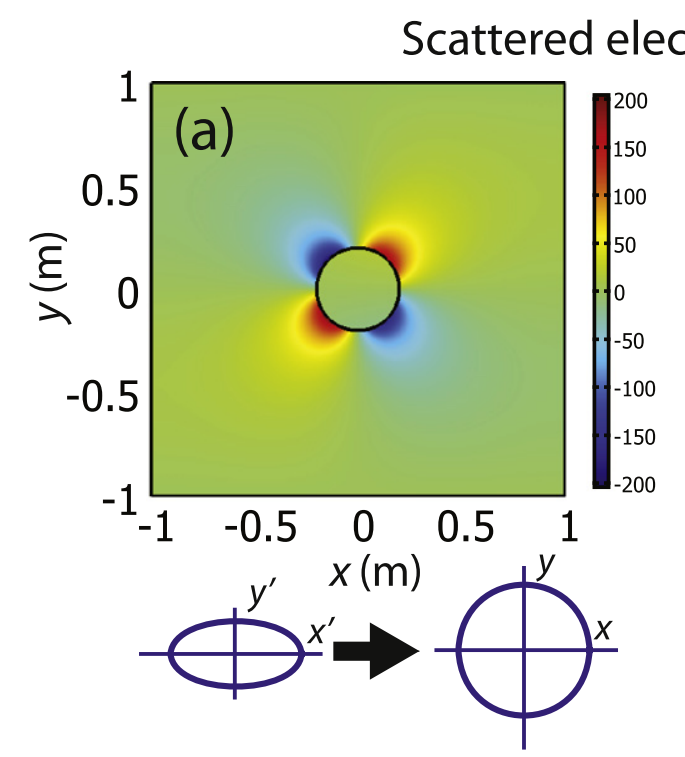

ctric field $E_{x}(\mathrm{~V} / \mathrm{m})$
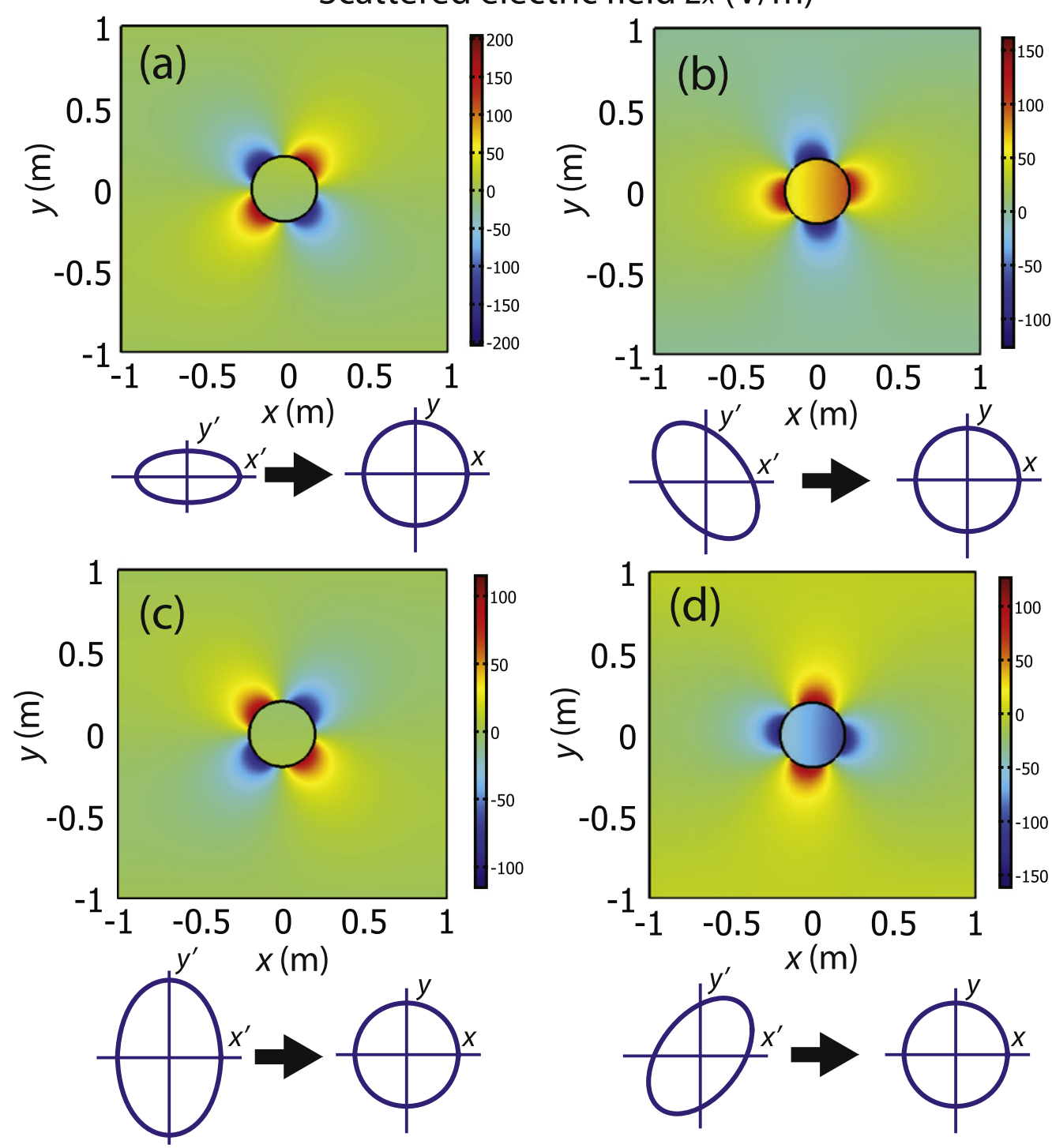

Figure 1. Full-wave simulation of the rotation of the scattering pattern by the angle (a) $\beta=0$, (b) $\beta=\pi / 2$, (c) $\beta=\pi$, and (d) $\beta=3 \pi / 2$ (radius $a=0.2 \mathrm{~m}$, polarizability $\alpha_{e}=0.01 \mathrm{~m}^{2}$, wavelength $\lambda=6 \mathrm{~m}, A=1 \mathrm{~V} / \mathrm{m}, \varepsilon^{\prime}=1, \mu^{\prime}=1$ ). Incident wave propagates from left to right. The bottom of each density plot shows the transformation of the ellipse in virtual space $\left(x^{\prime}, y^{\prime}\right)$ to the circle in physical space $(x, y)$.

field resulting from the change of electric field are negligible and we can keep the value of function $\psi_{3}$ unaltered. In the exact theory the change of the electric field would require the change of the magnetic field and $\psi_{3}$ as well. In figure 2(a) we demonstrate how the above proposition works for the potential function $\psi_{2}(r, \varphi, z)=r[\sin \varphi-B \sin (2 \varphi)]$. The predicted dependence $E_{x}^{(\mathrm{sc})} \sim \sin (3 \varphi)$ is well-observed in figure 2(a). The coordinate transformation transfers the contour of the vacuum particle in virtual space into the cylinder in physical space as shown in figure 2(c). Quasistatic scattered field is no longer observed when the radiation wavelength is comparable to the radius of the cylinder (see figure 2(b)). Indeed, in this case, the magnetic scattered field is noticeable and the phases at particle's center and cylinder's interface cannot be treated equal. The boundary conditions cannot be solved in the way as it has been done before.

\section{Predicting optical forces}

What one really needs in opto-mechanical applications is to control the direction and magnitude of the optical force. In this section we show the prospect of getting an arbitrary force using transformation optics.

The previous section has been ended up with the statement that any function $\psi_{2}$, therefore arbitrary quasistatic electric field at the interface, can be obtained. Thus we have the whole set of three potential functions, one of which is arbitrary, and can expect that the optical force calculated using (10) can exhibit many possibilities. This force has an approximate character and therefore well works only if its value is quite great, when we can safely neglect the scattered magnetic field. 

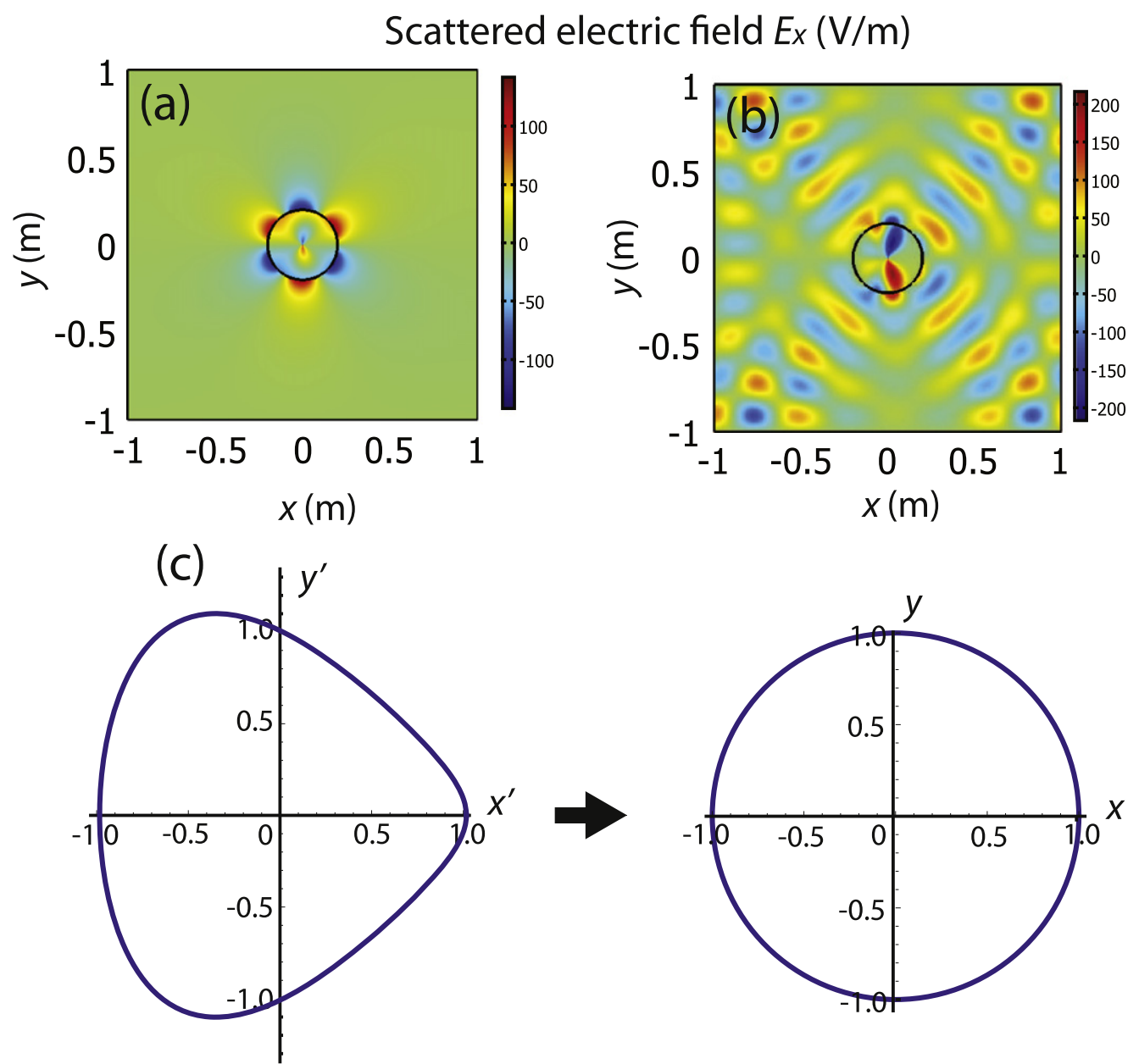

Figure 2. (a) Distribution of the scattered electric field $E_{x}$ specified by the potential function $\psi_{2}(r, \varphi, z)=r(\sin \varphi-0.25 \sin (2 \varphi))$ at the wavelength (a) $\lambda=6 \mathrm{~m}$ and (b) $\lambda=0.3 \mathrm{~m}$ (radius $a=0.2 \mathrm{~m}, A=1 \mathrm{~V} / \mathrm{m}, \varepsilon^{\prime}=1, \mu^{\prime}=1$ ). Incident wave propagates from left to right. (c) Coordinate transformation of the region in virtual space $\left(x^{\prime}, y^{\prime}\right)$ into the circle in physical space $(x, y)$.

Taking the ansatz of the potential function as

$$
\begin{aligned}
\psi_{2}(\boldsymbol{r})= & r\left(\sin \varphi+B_{1} \sin \left(\varphi+\beta_{1}\right)\right. \\
& \left.+B_{2} \sin \left(2 \varphi+\beta_{2}\right)+B_{3} \sin \left(3 \varphi+\beta_{3}\right)\right)
\end{aligned}
$$

the two non-zero components of the optical force per unit cylinder's length can be written as follows

$$
\begin{aligned}
& f_{x}=-\frac{A^{2} a}{8}\left(B_{1} B_{2} \cos \left(\beta_{1}-\beta_{2}\right)+3 B_{2} B_{3} \cos \left(\beta_{2}-\beta_{3}\right)\right), \\
& f_{y}=-\frac{A^{2} a}{8}\left(B_{1} B_{2} \sin \left(\beta_{1}-\beta_{2}\right)+3 B_{2} B_{3} \sin \left(\beta_{2}-\beta_{3}\right)\right) .
\end{aligned}
$$

Since $\pi<\beta<2 \pi$ corresponds to the change of sign of coefficient $B$, we assume $B>0$. The force does not vanish, when the potential function has two terms of the form $B_{n} \sin \left(n \varphi+\beta_{n}\right)$ and $B_{n+1} \sin \left((n+1) \varphi+\beta_{n+1}\right)$, i.e. $B_{1}$ and $B_{2}$ or $B_{2}$ and $B_{3}$ in (27).

It is curious that though the incident plane wave propagates along the $x$ axis, the optical force can be arbitrarily directed in the cross-section of the cylinder. For $B_{3}=0$, the direction is fully determined by the difference of the angles $\beta_{1}$ $-\beta_{2}$. For example, forward force $\left(f_{x}>0, f_{y}=0\right)$, backward force $\left(f_{x}<0, f_{y}=0\right)$, and orthogonal force $\left(f_{x}=0, f_{y}<0\right)$ appear at $\beta_{1}-\beta_{2}=\pi, \beta_{1}-\beta_{2}=0$ and $\beta_{1}-\beta_{2}=\pi / 2$, respectively. In figure 3 , we show the regions in virtual space that are transformed into the circle in physical space. When the force is not directed along the $x$ axis, the regions lose their symmetry. Indeed, the unconventional direction of the force requires that the rotation of the field which properly deforms the region to be transformed to the circle.

The angle-dependence of the dielectric permittivity and magnetic permeability tensors calculated according to equation (4) is quite complicated (see figure 4) for the case of pulling force $\boldsymbol{f}=-\left|f_{x}\right| \boldsymbol{e}_{x}$. The off-diagonal component $\varepsilon_{r \varphi}$ takes both positive and negative values, while the rest of the components oscillate near unity. One more drawback of such a tensor is the singularity at cylinder's center (angle dependence cannot be observed at one point). Core-shell particles should be considered to overcome this problem.

\section{Discussion and conclusion}

The results obtained above can be straightforwardly generalized to the cases of arbitrary incident field and spherical 

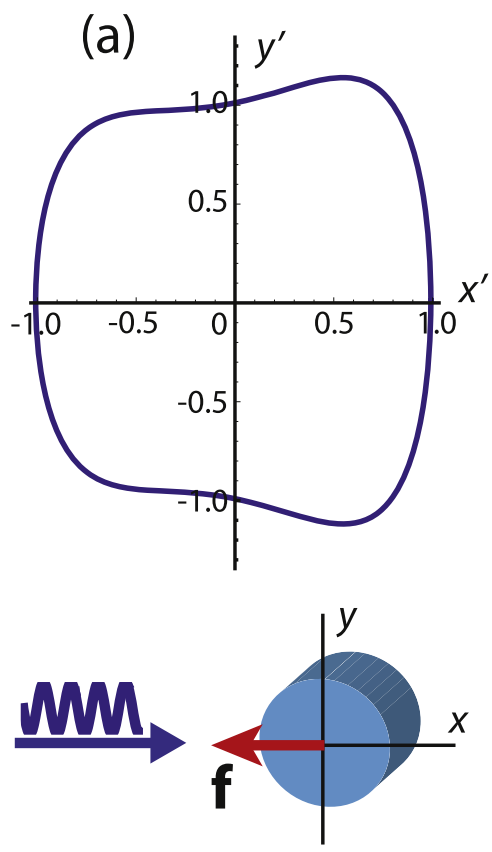
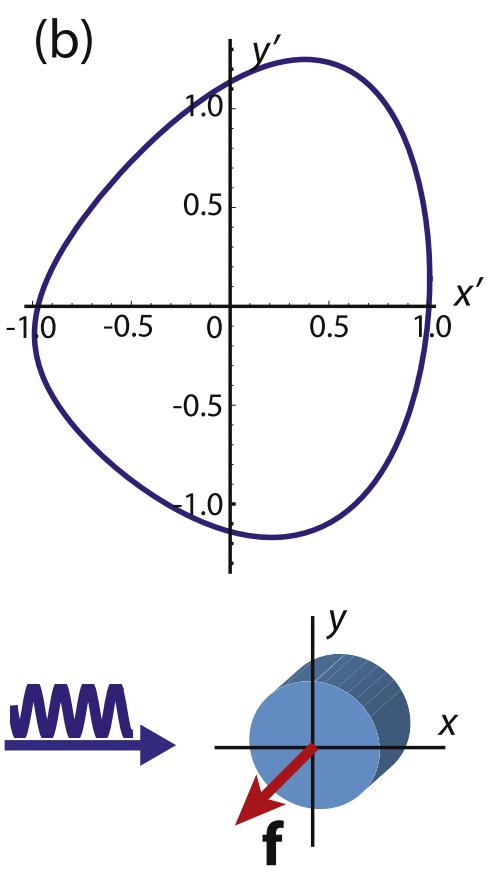
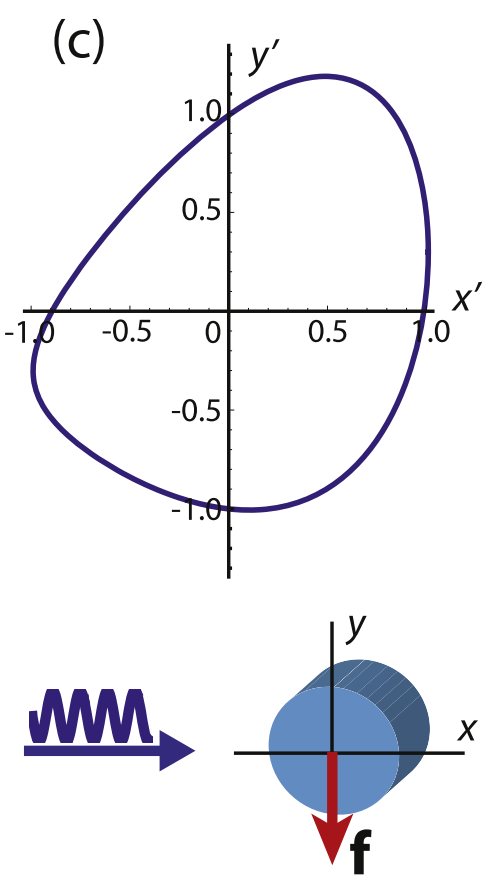

Figure 3. Region of transformation in virtual space (top row) and direction of the force $f$ exerted by the $x$-incident plane wave (bottom row) for three sets of parameters in potential function (27): (a) $B_{1}=B_{3}=0.2, B_{2}=0.1$, and $\beta_{1}=\beta_{2}=\beta_{3}=0$; (b) $B_{1}=B_{2}=0.2, B_{3}=0$, and $\beta_{1}=\pi / 2 \beta_{2}=\beta_{3}=0$; (c) $B_{1}=0.3, B_{2}=0.2, B_{3}=0, \beta_{1}=\pi / 2$, and $\beta_{2}=\beta_{3}=0$.

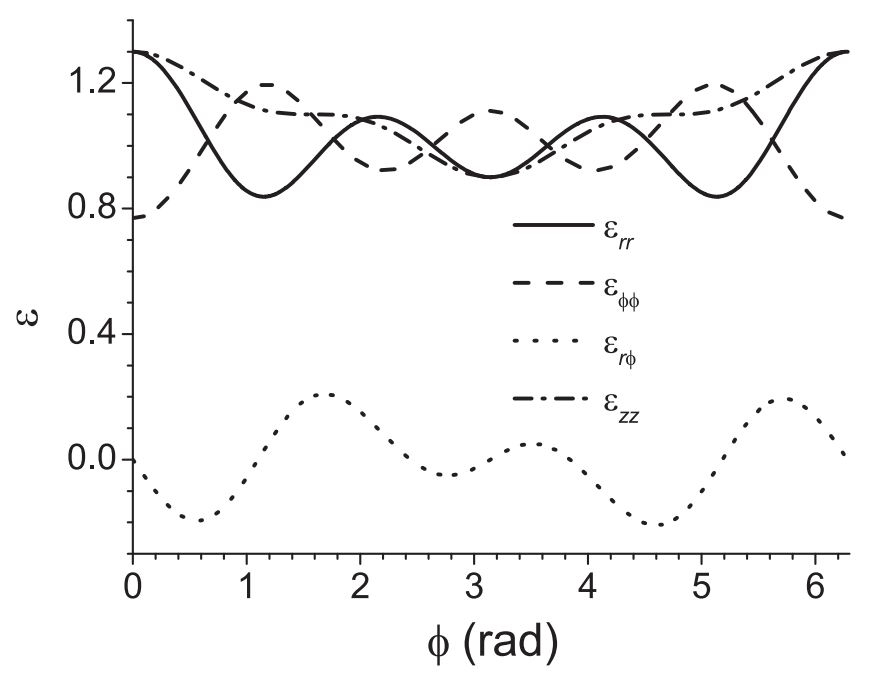

Figure 4. Angle dependence of the components of dielectric permittivity tensor $\hat{\varepsilon}=\hat{\mu}$ for $B_{1}=B_{2}=0.1, B_{3}=0$, and $\beta_{1}=\beta_{2}=\beta_{3}=0$.

particles in the approximation of small size of the particle compared with the wavelength. The rotation matrix for the sphere is determined by the angle and the axis of rotation; therefore, scattering pattern and the optical force are rotated in the three-dimensional space. Scattered magnetic field can be also designed in quasistatic regime.

The case of the particle sizes comparable to the wavelength is much more complicated. In this case, we can also choose arbitrary scattered electric field at the interface as for quasistatic fields, but the scattered magnetic field cannot be arbitrary then. In fact, they are connected by Maxwell's equations or, equivalently, with the surface impedance at the interface between the particle and ambient medium $\hat{\Gamma}$. Hence the specified scattered electric field $\left.\boldsymbol{I E}\right|_{S_{\text {sc }}}$ (e.g., defined by the potential functions $\psi_{1}$ and $\psi_{2}$ ) must result in the magnetic field $\left.\boldsymbol{I} \boldsymbol{H}\right|_{S_{\text {sc }}}$ (potential function $\psi_{3}$ ), because they are connected via the surface impedance as $\left.\boldsymbol{I} \boldsymbol{E}\right|_{S_{\mathrm{sc}}}=\left.\hat{\Gamma} \boldsymbol{I} \boldsymbol{H}\right|_{S_{\mathrm{sc}}}$. The surface impedance technique has been developed, e.g., in $[24,25]$, where the impedances of the partial cylindrical and spherical waves can be found. Thus, the potential functions and material tensors should depend on the solutions of the Maxwell equations (Hankel functions of argument $\omega a / c$ ). This inevitably leads to the lossy dispersive transformation media.

Transformation optics predicts the pulling optical force for an object made of anisotropic inhomogeneous materials. Pulling force makes a reality the tractor beams recently proposed in a number of works [26-29]. The transformation optics approach is advantageous for searching material parameters of the objects that can be pulled by a simple wave, such as a plane wave, in contrast to the previous approaches dealing with intricate light beams. Moreover, the transformation optics paves the way to the total control of the optical force. With an impenetrable cavity inside the transformationmedium particle (as for a cloak), any object inside the cavity can be dragged in any direction by a simple plane wave.

The main problem with the experiment is the fabrication of the particles with required material tensors, e.g., as in figure 4 . If the scatterers are manufactured, their characterization can be carried out, e.g., using the dark-field microscopy as in [16]. However, we do not discuss the required experimental facilities here. Current research is destined to show one more path for the transformation optics. 
In conclusion, we have applied the method of transformation optics to the prediction of the scattered fields and optical forces in quasistatic regime. We have revealed that the coordinate transformation can be used to generate any scattered electric field. Unlike the ordinary transformation optics devices, which keep the boundary of the device upon transformation from virtual to real space, the scattered field generators change the transformation region. This very feature is responsible for the creation of the electromagnetic field, which is absent in the virtual space. Our approach perfectly controls the optical forces, thus being good tool in optical micromanipulation.

\section{Acknowledgments}

Financial support from the Grant of the President of the Republic of Belarus is acknowledged.

\section{References}

[1] Pendry J B, Schurig D and Smith D R 2006 Controlling electromagnetic fields Science 312 1780-3

[2] Leonhardt U 2006 Optical conformal mapping Science 312 1777-80

[3] Schurig D, Mock J J, Justice B J, Cummer S A, Pendry J B, Starr A F and Smith D R 2006 Metamaterial electromagnetic cloak at microwave frequencies Science 314 977-80

[4] Leonhardt U and Philbin T G 2009 Geometry and light: the science of invisibility Prog. Opt. 53 69-152

[5] Cai W, Chettiar U K, Kildishev A V and Shalaev V M 2007 Non-magnetic cloak without reflection Nat. Photonics 1 224-6

[6] Rahm M, Schurig D, Roberts D A, Cummer S A, Smith D R and Pendry J B 2008 Design of electromagnetic cloaks and concentrators using form-invariant coordinate transformations of Maxwells equations Photon. Nanostruct.: Fundam. Applic. 6 87-95

[7] Li J and Pendry J B 2008 Hiding under the Carpet: a new strategy for cloaking Phys. Rev. Lett. 101203901

[8] Yaghjian A D and Maci S 2008 Alternative derivation of electromagnetic cloaks and concentrators New J. Phys. 10 115022

[9] Chen H and Chan C T 2007 Transformation media that rotate electromagnetic elds Appl. Phys. Lett. 90241105

[10] Luo Y, Chen H, Zhang J, Ran L and Kong J A 2008 Design and analytical fullwave validation of the invisibility cloaks, concentrators, and eld rotators created with a general class of transformations Phys. Rev. B 77125127

[11] Lai Y, Ng J, Chen H, Han D Z, Xiao J J, Zhang Z-Q and Chan C T 2009 Illusion Optics: the optical transformation of an object into another object Phys. Rev. Lett. 102253902

[12] Li C, Meng X, Liu X, Li F, Fang G, Chen H and Chan C T 2010 Experimental realization of a circuit-based broadband illusion-optics analogue Phys. Rev. Lett. 105233906

[13] Luo Y, He L-X, Wang Y, Chan H L W and Zhu S-Z 2011 Changing the scattering of sheltered targets Phys. Rev. A 83 043809

[14] Novitsky A V, Zhukovsky S V, Barkovsky L M and Lavrinenko A V 2012 Field approach in the transformation optics concept Progress In Electromagnetics Research 129 485-515

[15] Kerker M, Wang D and Giles G 1983 Electromagnetic scattering by magnetic spheres J. Opt. Soc. Am. 73 765-7

[16] Fu Y H, Kuznetsov A I, Miroshnichenko A E, Yu Y F and Luk'yanchuk B 2013 Directional visible light scattering by silicon nanoparticles Nat. Commun. 41527

[17] Kuznetsov A I, Miroshnichenko A E, Fu Y H, Zhang J B and Luk'yanchuk B 2012 Magnetic light Sci. Rep. 2492

[18] Luk'yanchuk B S, Voshchinnikov N V, Paniagua-Domnguez R and Kuznetsov A I 2015 Optimum forward light scattering by spherical and spheroidal dielectric nanoparticles with high refractive index ACS Photonics 2 993-9

[19] Ashkin A et al 1986 Observation of a single-beam gradient force optical trap for dielectric particles Opt. Lett. 11 288-90

[20] Grier D G 2003 A revolution in optical manipulation Nat. Photonics 424 810-6

[21] Dholakia K and Čižmár T 2011 Shaping the future of manipulation Nat. Photonics 5 335-42

[22] Novitsky A V 2011 Inverse problem in transformation optics J. Opt. 13035104

[23] Jackson J D 1998 Classical Electrodynamics (New York: John Wiley) p 611

[24] Novitsky A V and Barkovsky L M 2005 Operator matrices for describing guiding propagation in circular bianisotropic fibres J. Phys. A.: Math. Gen. 38 391-404

[25] Novitsky A and Barkovsky L 2008 Matrix approach for light scattering from a multilayered rotationally symmetric bianisotropic sphere Phys. Rev. A 77033849

[26] Chen J, Ng J, Lin Z and Chan C T 2011 Optical pulling force Nat. Photon. 5 531-4

[27] Novitsky A, Qiu C-W and Wang H 2011 Single gradientless light beam drags particles as tractor beams Phys. Rev. Lett. 107203601

[28] Sukhov S and Dogariu A 2011 Negative nonconservative forces: Optical tractor beams for arbitrary objects Phys. Rev. Lett. 107203602

[29] Ruffner D B and Grier D G 2012 Optical conveyors: a class of active tractor beams Opt. Express 109163903 